\title{
A Typology of Evidence Based Practice Research Heuristics for Clinical Laboratory Science Curricula
}

\author{
ELIZABETH K LEIBACH, BARBARA L RUSSELL
}

\begin{abstract}
A typology of EBP research heuristics was defined relative to clinical laboratory science levels of practice. Research skills requisite for CLS baccalaureate level are associated mainly with quality control of analytic processes. Research skills at master's level are associated with pre- and post-analytic investigations, as well. Doctoral level CLS practice portends to utilize research skills facilitating quality investigations at the systems level.
\end{abstract}

ABBREVIATIONS: ASCLS = American Society for Clinical Laboratory Science; $\mathrm{CTR}=$ clinical and translational research; CLS = clinical laboratory science/clinical laboratory scientists; DCLS = doctorate in clinical laboratory science; $\mathrm{EBM}=$ evidence based medicine; $\mathrm{EBP}$ = evidence based practice

INDEX TERMS: clinical and translational research; clinical and translational science; clinical doctorate; clinical laboratory science; clinical outcomes; critical thinking; epidemiology; evidence based practice; expert practice; professional doctorate; research methods.

Clin Lab Sci 2010;23(3)Suppl:3-46

Elizabeth Kenimer Leibach, Ed.D., MLS ${ }^{C M}, S B B$, Clinical Laboratory Science, Medical College of Georgia, Augusta, Georgia 30912-0500

Barbara Landis Russell, Ed.D., MT, SH, Clinical Laboratory Science, Medical College of Georgia, Augusta, Georgia 30912-0500

Corresponding Author: Elizabeth Kenimer Leibach, Ed.D., MLS ${ }^{C M}$, SBB, Department of Biomedical and Radiological Sciences, EC 2437, 987 St. Sebastian Way, Medical College of Georgia, Augusta, Georgia 309120500,(706)721-3046, ekenimer@mcg.edu

\section{INTRODUCTION}

The emerging view of evidence based practice in clinical laboratory science is one with patient-centered focus and interaction. In venues in which the impact of laboratory information is determined to impact patients' well-being, clinical laboratory scientists, functioning at levels of practice appropriate to their education in clinical research, will collate, interpret, and summarize clinical laboratory information and consult with patients and other healthcare providers to optimize services delivery and desirable health outcomes. ${ }^{1 .}$

Working backward from this prototype practice to CLS educational programs, didactic coursework and internships must be designed to inform practice and expose students to clinical experiences providing the greatest opportunity to develop the research skills necessary not only to utilize evidence in clinical decision-making but also to generate and communicate data-supported practice guidelines, to monitor patients' critical paths, to evaluate and introduce new technology, to develop quality indicators, and to create and analyze testing algorithms. Not only will this CLS evidence be used in clinical decision-making, but these ordering and utilization data can be analyzed to support development of best practices to decrease errors, increase patient safety, and communicate CLS evidence for practice improvement across all healthcare delivery systems, public and private. Though the need for this CLS-specific clinical and translational research (CTR) has been clearly articulated, educational strategies for teaching the tenets of EBP appropriate for each level of CLS practice have not been previously defined. ${ }^{2}$.

\section{RESEARCH AIMS}

Literature related to the theoretical underpinnings of CLS EBP was explored. In 2002, critical thinking behaviors, emerging from CLS practice, were reported 


\section{RESEARCH AND REPORTS}

from a large national survey of CLS practitioners. Common themes, or CT factors, were then distilled from the CT behaviors identified. ${ }^{3}$ These CT factors have been shown to be exhibited in expert practice in the healthcare professions, and can be considered categories of observable expert practice behaviors emanating from the critical thinking process. ${ }^{4,5}$ Further, the epidemiologically-based methodologies of CTR can be shown to serve as an important applied platform for the exercise of critical thinking behaviors comprising expert practice. These CT-expert practice-EBP connections are discussed in more detail elsewhere. ${ }^{3}$ EBP heuristics needed to effectively apply methodology of CTR in CLS practice can be identified and ordered according to extent of CT behaviors involved. The aim of this study was to develop a research skills typology defining component constructs of EBP to be taught at CLS baccalaureate, master's, and doctoral practice levels.

\section{EVIDENCE BASED PRACTICE OVERVIEW}

The larger Evidence Based Medicine (EBM) movement, from which DCLS practice tenets are derived, is driven by computerization (and information overload), the need for cost-efficiency, and public demand for best treatment options. ${ }^{6}$ EBM is commonly described using the definition of the author of the movement, David Lawrence Sackett, Canadian-born, American-educated physician:

"The conscientious, explicit, and judicious use of the best evidence in making decisions about the care of individual patients." 7

Since the inception, however, much literature and many processes have been developed to support the generation and interpretation of "best evidence" and "making decisions about the care of individual patients." A quick search will reveal a plethora of options for EBM study. Indeed, most health professions have adapted Sackett's concepts to their own disciplines, and after this adaptation, the concept and practice strategy becomes "Evidence Based Practice" (EBP). ${ }^{8}$ Regardless of the adapted professional specificity, EBP shares with EBM a process for generating, evaluating, and utilizing best evidence in professional decision-making. This process denoted as "EBM Fundamentals" is provided in Figure $1 .{ }^{7}$
The emerging view of CLS EBP is one with patientcentered focus and interaction. In venues in which the impact of laboratory information is determined to impact patients' well-being, CLS practitioners collate, interpret, and summarize clinical laboratory information and consult with patients and other healthcare providers to optimize services delivery and desirable health outcomes. This focus of quality assurance is shared with traditional EBM practice, i.e., effectiveness, as defined below, and encompasses patient diagnoses, therapy monitoring, and assessing clinical outcomes. In addition, CLS EBP must include the traditional measures of quality (quality control) as well as quality evaluation, assurance, and improvement at the systems level, where the EBP of other disciplines supported by laboratory information integrate with CLS EBP. An axiom borrowed from the manufacturing sector figures prominently in the measurement of quality of clinical laboratory information: "Good quality costs less than poor quality." Thus, EBP for CLS should incorporate clinical studies resulting in, for example, diagnostic testing descriptive studies, practice guidelines development, and cost-efficiency analysis as well as conventional effectiveness assessments like clinical trials and observational studies. ${ }^{10}$ These additional areas of practice are defined, in quality terms, as efficacy, e.g., diagnostic testing studies, practice guidelines development, and efficiency, e.g., cost-efficiency analysis. ${ }^{11}$ These quality definitions meaningful in CLS EBP are as follows:

- Efficacy measures how well a treatment works in clinical trials or laboratory studies, while

- Effectiveness relates to how well a treatment works in practice.

- Efficiency is broadly defined as "the mix of health care resource inputs that produce optimal quantity and quality of health and health care outputs... among individual, institutional, and groups of providers." 11

The goal of the CLS EBP effort is to provide quality healthcare in the most cost-effective way. And it follows that quality healthcare delivery protocols, guidelines, and processes should be supported by evidence from rigorously constructed clinical studies that are experimental or observational in design. CLS practice must address all areas of quality services delivery: 
efficacy, effectiveness, and efficiency. In other words, CLS EBP must address the entirety of the diagnostic and therapeutic processes that are supported by CLS information: quality in pre-analytic and post-analytic aspects of services delivery, as well as assuring quality of analytic processes.
EBM Fundamentals...

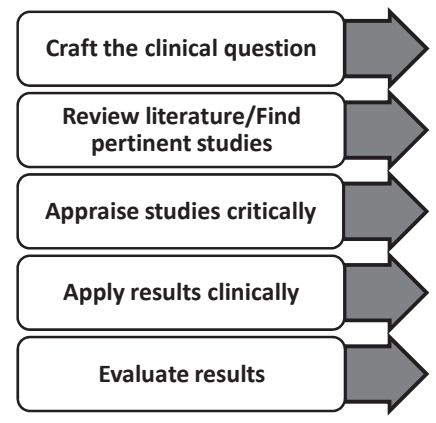

CLS EBP Fundamentals...

\begin{tabular}{|c|}
\hline $\begin{array}{c}\text { Assess CLS quality indicators } \\
\text { Identify pivotal diagnoses or } \\
\text { procedures (tests) }\end{array}$ \\
\hline $\begin{array}{c}\text { Search for practice guidelines / } \\
\text { standards }\end{array}$ \\
\hline $\begin{array}{c}\text { Compare existing critical paths } \\
\text { to guidelines/standards }\end{array}$ \\
\hline Evaluate downstream effects of \\
differences
\end{tabular}

Figure 1. Comparison of EBM and CLS EBP Processes.

EBP, then, can be succinctly stated as:

"The conscientious, explicit, and judicious use of the best evidence from CLS in making decisions about the care of individual patients."

\section{EBP RESEARCH HUERISTICS TYPOLOGY FOR CLS EDUCATION}

EBP is clinical in nature with roots in epidemiological methodology. As such, these clinical and translational approaches are not typically addressed to any significant degree in CLS curricula. Certainly, the application of EBP research methodologies in CLS practice has never been acknowledged nor have EBP research skills been indexed to CLS practice levels. Common components of EBP research are listed in Table 1. Figure 2. summarizes an instructional typology suggesting an ordered presentation of clinical research constructs from more basic (baccalaureate) to complex (doctorate) in CLS education. EBP constructs appropriate for the CLS baccalaureate level are those statistical concepts underlying quality control assessment and concepts related to critical appraisal of the literature. Appropriate for the CLS master's level are instruction in human and non-human research policy, design, and methodology as well as practice in identification and utilization of evidence to evaluate analytic testing phase questions. At the doctoral level, EBP instruction should focus on clinical and translational research concepts employed in effectiveness studies for evaluation of pre- and postanalytic testing phase questions.

Practice responsibilities at all practice levels should capitalize on these expanded scopes of knowledge. Evaluation of the analytic phase of the diagnostic testing process, i.e., quality control, is appropriate for the baccalaureate-prepared practitioner. Additional EBP knowledge at the master's level prepares these postbaccalaureate practitioners to identify and address clinical questions related to testing process efficacy and clinical effectiveness. These types of analyses can be categorized under the rubric of quality assurance. ${ }^{12}$ Doctorate-prepared practitioners should focus on clinical questions involving the appropriateness and utilization of CLS information. These doctoral-level analyses represent system-wide approaches to the assessment of clinical quality improvement questions involving the interpretation and utilization of laboratory information by multiple disciplines. ${ }^{1,8}$

\section{EBP RESEARCH MODELS IN CLS EDUCATION}

Much literature has been devoted to theoretical and practical aspects of statistical analysis of quality in laboratory testing. ${ }^{9}$ Further, statistical underpinnings as well as clinical applications of quality control are taught and modeled during associate and baccalaureate preservice CLS education. These elements of practice and quality management are the foundations of EBP in master's and doctoral level CLS education and practice. Types of research designs appropriate for master's level CLS education and practice and examples of completed master's level projects are listed in Table 2.

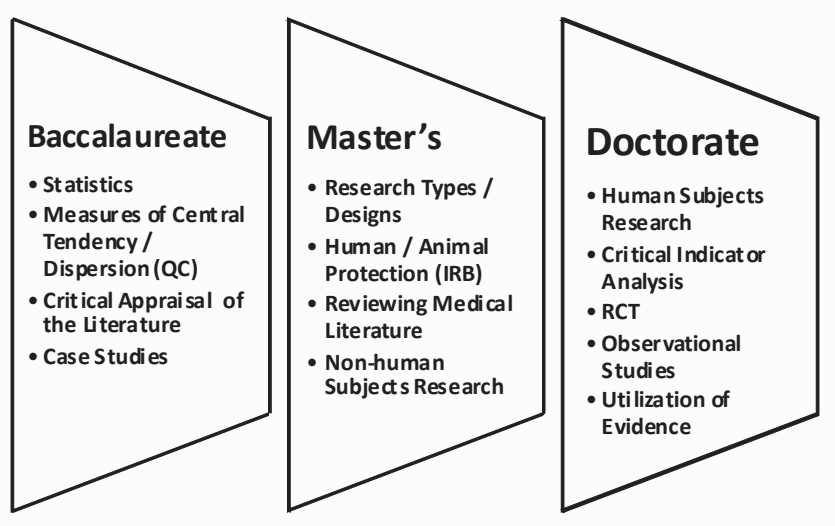

Figure 2. EBP Research Typology for CLS Education 


\section{RESEARCH AND REPORTS}

At the CLS doctoral level (DCLS), the complexity of $\mathrm{EBP}$ research and commensurate requisite education increases due to the systems approach required in clinical investigations as well as the interactive nature of CLS EBP and the EBP of other healthcare disciplines. As an example of this increasing complexity, suppose the results of the master's level project correlating the five-part white cell differential to total white count in leukemia patients suggested a substantive change in ordering patterns for that diagnosis group. First, an EBP study testing this hypothesis would have to be designed, using the EBP process, to identify and assess patient outcomes with the potential for variance relative to these hematological parameters. After the study was implemented and evaluated and if the hypothesis were accepted, practice guidelines could be altered and new guidelines constructed and proposed. Acceptance of these new practice guidelines by the medical staff would not only alter CLS EBP but would also impact EBP of physicians, nurses, and other healthcare providers involved in the critical path of patients in this diagnosis group.

Table 1. EBP Research Component Constructs

- Statistical Analyses

- $\quad$ Bayes' Theorum; Causation; Hypothesis Testing

- Sensitivity/Specificity; ROC

- Predictive Value

- Experimental Research Design

- Diagnostic Testing (Screening vs. Specific; Incremental Gain; Bias)

- Strength of Evidence

- Randomized Clinical Trials (RCT)

- Observational Studies

- Outcomes / Cost-effectiveness Analyses / Cohort / Cross-sectional

- Practice Guidelines Development / Clinical Prediction Rules

- Meta-analysis

Like the EBP process itself, the choice of DCLS EBP projects to pursue should be data-driven. Given that the roots of CLS EBP are embedded in quality, projects should be considered that offer the greatest potential impact on quality services delivery. Among that population would be diagnoses impacting laboratory operations because of their frequency, increased risk of errors in ordering or interpreting test results, or high per unit laboratory direct costs. The following steps should

Table 2. Examples of Master's Level EBP Research Designs and Projects

- The value of gram stains in the identification of positive sputum cultures and in the diagnosis of bacterial pneumonia

- Effects of hemolyzed specimens on time and cost of laboratory testing at a large medical center

- Specimen labeling errors in the Transfusion Service: A Q-probe analysis of contributing factors based upon rate

- Determining the need to perform a five-part WBC differential count based in its correlation to the change in the TLC

- A performance evaluation and optimization based in suspect flags derived from seven hematologic parameters obtained from the Sysmex XE-2100

- Prevalence of HPC and Chlamydia trachomatis in African American women may lead to cervical and breast cancer

- Sensitivity and specificity of anti-RAGE and $A \beta$ immunoglobulin assay in patients with mild cognitive impairment versus severe cognitive impairment

- The effect of phlebotomy education on specimen labeling errors: A comparative study

guide the development and utilization of evidence for performance improvement at the systems level: 1,6,8,9,12

- Identify collaborating healthcare subspecialties and services utilizing and monitoring significant clinical laboratory information for medical decisionmaking, e.g., pharmacy, quality improvement, therapeutics/transfusion service, infectious disease, risk management, utilization review, internal medicine, elective surgery, family medicine, emergency medicine, pediatrics, geriatrics, oncology, hospital medicine.

- Identify high risk, high frequency, and high cost procedures and diagnoses as well as quality indicators in each of the subspecialties and services. The procedures and diagnoses identified as well as quality indicators chosen to be monitored for institutional quality assessment will provide the criteria for determining study type and focus, i.e., outcome/diagnosis study, cost-effectiveness analysis, practice guideline development/comparison.

- Identify high risk, high frequency, and high cost clinical laboratory tests, procedures, and/or diagnoses in the clinical laboratory as well as related quality indicators for each of the subspecialties.

- Compare high risk, high frequency, and high cost tests, procedures, diagnoses, and quality indicators 


\section{RESEARCH AND REPORTS}

in the subspecialties to those in CLS. Apply evidence based techniques to investigate patient cases related to procedures, diagnoses, and quality indicators common to both. Comparison of clinical laboratory quality indicators to those diagnoses/procedures with high clinical laboratory resources impact will provide criteria for determining diagnoses/procedures for study focus.

- Analyze utilization data from focused clinical study(ies) to propose common steps and strategies in design and development of representative evidence based study models that can be implemented in DCLS internship experiences and, afterwards, in practice.

The role of the CLS practitioner would be to apply EBM process steps to their appropriate level of CLS practice by: (1) evaluating projects with potential for quality impact, i.e., those related to assessment of quality indicators, efficacy, effectiveness, and/or efficiency, (2) identifying projects related to diagnoses and/or procedures (tests) with greatest impact on quality services delivery, (3) searching the literature for practice guidelines and standards relevant to these projects, (4) comparing existing internal critical paths associated with these projects to relevant published guidelines and standards, and (5) evaluating the downstream effects of differences in internal practices and relevant published protocols. The CLS EBP process and its relationship to the EBM process model are summarized in Figure 1.

\section{SUMMARY AND CONCLUSION}

In preparation for their advanced practice roles, CLS students will perform clinical internship duties that will prepare them for practice upon program completion. Baccalaureate students, and baccalaureate-prepared CLS practitioners later, will control laboratory analytic processes in all venues where testing is performed. Master's students, and master's-prepared CLS practitioners, will address issues of quality related to assessment of effectiveness of laboratory processes and information. DCLS students, and DCLS practitioners, will collate, summarize, and present laboratory information related to patients and intervention groups representing significant clinical laboratory resource utilization. The typology of research skills presented here provides the epistemological frame for defining, teaching, and applying this EBP process in CLS.

\section{REFERENCES}

1. Practice Levels and Educational Needs for Clinical Laboratory Personnel. American Society for Clinical Laboratory Science 2009: http://www.ascls.org/position/LevelsofPractice.asp. Accessed 2010, March 15.

2. Translational Research Working Group. National Cancer Institute. Available from_http://www.cancer.gov/trwg/TRWGdefinition-and-TR-continuum and http://ctsaweb.org/ upload edfiles/Welcome_2-9-20072.ppt\#258,4k, Definition of TranslationalResearch. Accessed 2010, March 15.

3. Kenimer, E. A. The identification and description of critical thinking behaviors in the practice of clinical laboratory science, part 1: Design, implementation, and evaluation of a national survey. Journal of Allied Health 2002; 31(2), 56 - 63.

4. Benner P. From Novice to Expert: Excellence and Power in Nursing Practice. Menlo Park: Addison-Wesley, 1984.

5. Lifelong Learning in Medicine and Nursing. American Association of Colleges of Nursing 2010: http://www. aacn.nche.edu/Education/pdf/MacyReport.pdf. Accessed 2010, March 15.

6. McQueen, M. J. Overview of evidence-based medicine: Challenges for evidence-based laboratory medicine. Clinical Chemistry 2001; 47: 1536-46.

7. Sackett, D., Rosenberg, W. M., Gray, J., Haynes, R. B., \& Richardson, W. S. Evidence-based medicine: What it is and what it is not. British Medical Journal 1996; 312, 71-2.

8. Leibach, E. K. The doctorate in clinical laboratory science: A view of clinical practice development. Clinical Laboratory Science 2008; 21(4), 196-8.

9. Westgard, J. O. Six Sigma Quality Design and Control (2 ${ }^{\text {nd }}$ ed.). Madison, WI: Westgard Quality Corporation; 2006.

10. Price, C. Evidence based laboratory medicine. SciTopics, August 25, 2008. Available from: http://scitopics.com/ Evidence_Based_Laboratory_Medicine.html. Accessed 2010, March 15

11. Institute of Medicine 2006. "Performance Measurement: Accelerating Improvement" in Pathways to Quality Health Care series from http://www.nap.edu/openbook.php?isbn=0309 100070. Accessed 2010, March 15.

12. Leibach, E. K. The doctorate in Clinical Laboratory Science: CLS education beyond the baccalaureate. Clinical Laboratory Science 2008; 21(2), 78-81. 